\title{
Analyse von Wirkungsgradverlusten in Kettenfördersystemen
}

\author{
Marcus Bona*, Jens Sumpf, Markus Golder \\ Professur Förder- und Materialflusstechnik, Technische Universität Chemnitz, Deutschland \\ * Korrespondenz: marcus.bona@mb.tu-chemnitz.de
}

Erhalten am 4. November 2020; Angenommen am 5. November 2020; Online zugänglich seit 7. Dezember 2020

(C) 2020 by M. Bona u.a. Dies ist ein Open-Access-Artikel, der unter der Creative Commons Attribution License (CC-BY 4.0) verbreitet wird, die die uneingeschränkte Nutzung, Verbreitung und Vervielfältigung in jedem Medium erlaubt, vorausgesetzt, das Originalwerk wird ordnungsgemäß zitiert.

\begin{abstract}
Gerade in der heutigen Zeit, vor dem Hintergrund steigender globaler Erwärmung, rückt die Energieeffizienz elektrisch angetriebener technischer Systeme immer mehr in den Fokus. So auch im intralogistischen Materialtransport. Der Gesamtwirkungsgrad eines Stetigförderers, also das Verhältnis aus aufgewandter elektrischer Leistung und der Transportleistung, ist dabei von der Effizienz seiner zahlreichen Einzelkomponenten abhängig. Bereits durchgeführte Messungen zeigen, dass dabei vor allem dem Antriebssystem große Bedeutung beikommt. Die in Kettenförderern am häufigsten eingesetzten Antriebe sind DrehstromAsynchron-Getriebemotoren. Diese weisen in ihrem Nennlastbereich zwar gute Wirkungsgrade von über $80 \%$ auf, werden im Großteil aller Fälle jedoch im unteren Lastbereich betrieben. Die oft viel zu groß ausgelegten Motoren arbeiten somit mit einem erheblich schlechteren Wirkungsgrad, was zu einem erhöhten Energieverbrauch führt. Der Beitrag befasst sich aus den genannten Gründen mit der Analyse von Wirkungsgradverlusten am Beispiel von Kettenförderern und geht im Speziellen auf antriebstechnische Komponenten wie Motor, Frequenzumrichter und Getriebe ein. Das Ziel ist die Einordnung dieser Verluste in den Gesamtwirkungsgrad eines Förderers, der u. a. Reibungs- und Hubanteile enthält. Weiterhin werden Schlussfolgerungen und Empfehlungen zur Verbesserung der Effizienz von Kettenförderern diskutiert.
\end{abstract}

KEYWORDS Kettenförderer, Wirkungsgrad, Energieeffizienz, Asynchronmotor, Reibwert

\section{Einleitung}

Das Auftreten von Verlusten beim Betrieb von Kettenförderern ist, wie in jedem anderen technischen System, nicht zu verhindern. Reibungsverluste, bei denen mechanische Energie in nicht mehr nutzbare Wärme umgewandelt wird, sind folglich bereits dem Arbeitsprinzip eines solchen Stückgutförderers geschuldet. Neben Reibungsverlusten z. B. zwischen Gleitkette und Gleitschiene, Lagerreibung oder Reibung im Zugmittel selbst, treten zudem auch Verluste in den elektronischen Komponenten wie Motoren oder Frequenzumrichter auf.

Gerade vor dem Hintergrund steigender Treibhausgasemissionen und dem damit verbundenen weltweiten Klimawandel, ist der energieeffiziente Betrieb von Förderanlagen innerhalb von Produktionseinrichtungen, eine große Herausforderung. Ziel des Beitrages ist 
es dementsprechend Kettenhersteller, Anlagenplaner und Anwender von Stetigfördersystemen in Bezug auf Wirkungsgradverluste ihrer Anlagen zu sensibilisieren, unnötige Energieverbräuche zu erkennen und zu senken, sowie diese bereits in der Planungsphase zu verhindern.

\section{Ausgangslage/Motivation}

Kettenförderer gehören zur Gruppe der Stetigförderer und dienen dem innerbetrieblichen Transport von gleichartigen Gütern zwischen einzelnen Stationen eines Logistiknetzwerkes. Einen großen Stellenwert im Bereich dieser ortsgebundenen Transportsysteme nehmen die Gleitkettenförderer ein. Aufgrund ihrer hervorragenden tribologischen Eigenschaften und geringer Massen werden die dabei verwendeten Gleitketten zunehmend aus Kunststoff gefertigt, was es dem Anwender erlaubt den Förderer schmiermittelfrei zu betreiben. Kunststoffgleitketten sind dadurch unter anderem für den Einsatz in sensiblen Bereichen wie z. B. Lebensmittel-, Pharma-, Verpackungsmittelindustrie prädestiniert. Ihr modularer Aufbau aus verschiedenen Streckenelementen (z. B. Gerade, Kurve, Steigung, Antrieb und Umlenkung) erlaubt es, den Förderer sehr flexibel an verschiedenste Förderaufgaben anzupassen. Abbildung 1 zeigt dafür zwei Beispiele, auf der linken Seite einen einsträngigen Förderer und rechts ein komplexes Beispiel aus der Getränkeindustrie, bei dem die Gleitketten mehrsträngig nebeneinander angeordnet sind.

Viele vorangegangenen Arbeiten zur Verbesserung der Energieeffizienz von Stetigförderern mit Ketten befassen sich allein mit der Reduzierung der Reibung in Förderanlagen [3, 4]. Für die Dimensionierung und Auslegung von Ketten und Antriebskomponenten ist der Reibwert zweifelsohne ein ausschlaggebender Faktor. Dass tribologische Verluste jedoch nur einen Anteil am Gesamtwirkungsgrad eines Kettenförderers darstellen, wird dabei oft vernachlässigt. Untersuchungen an Förderanlagen bei der Getränkeabfüllung haben beispielsweise gezeigt, dass die aufgenommene elektrische Wirkleistung des Motors in den Pausenzeiten, in denen die Ketten ohne Beladung und mit geringerer Geschwindigkeit
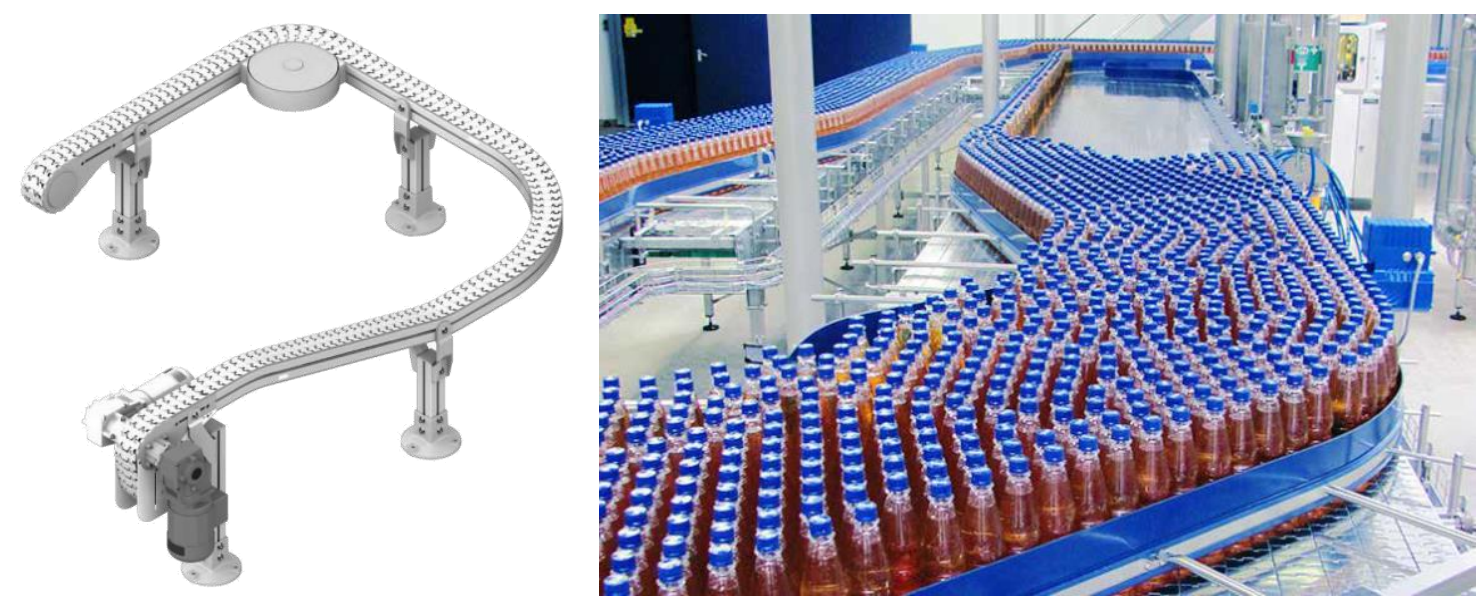

Abbildung 1: Förderlayout mit typischen Streckenelementen (links) [1] sowie Flaschentransport in der Getränkeindustrie (rechts) [2] 
laufen, um rund 12\% steigt. Das heißt entgegen der Erwartung verbraucht der Förderer bei voller Beladung und hoher Geschwindigkeit weniger Energie. Dieser Effekt lässt sich nur auf die Verluste im Antriebssystem, d.h. dem Motor, dem Getriebe und dem Frequenzumrichter, zurückführen.

Gängige Praxis bei der Auslegung und Auswahl von Antriebskomponenten bei Fördersystemherstellern ist eine grob geschätzte Annahme der benötigten Antriebsleistung. Die Berechnung des Bewegungswiderstandes und der daraus resultierenden Motorleistung erfolgen zumeist nicht. Um eventuelle Stillstände im Betrieb der Anlage zu vermeiden, wird der Antriebsmotor vorzugsweise überdimensioniert. Dass der Asynchronmotor dabei oft bei erheblich schlechteren Wirkungsgraden als vom Hersteller angeben arbeitet, ist häufig nicht bekannt oder wird toleriert.

\section{Wirkungsgradverluste in Kettenfördersystemen}

\subsection{Gesamtsystem}

Allgemein lässt sich der Gesamtwirkungsgrad eines Förderers $\eta_{\text {Ges }}$ nach Gleichung (1) als Verhältnis aus der umgesetzten mechanischen Leistung $P_{\text {mech }}$ und der dafür aufgewendeten elektrischen Leistung $P_{e l}$ berechnen.

$$
\eta_{\text {Ges }}=\frac{P_{\text {Transport }}}{P_{\text {el }}}
$$

Die elektrische Leistung kann dabei relativ einfach als Wirkleistung vor dem ersten Verbraucher, beispielsweise durch ein elektrisches Leistungsmessgerät, gemessen werden. Die Transportleistung dagegen ergibt sich aus der Umfangskraft $F_{U}$ des Zugmittels multipliziert mit der Fördergeschwindigkeit $v$, wobei $F_{U}$ dem Gesamtwiderstand aller Kräfte entspricht, die dem Zugmittel während des Transportes entgegenwirken. Sie beinhaltet somit alle Reibungswiderstände sowie Hub- und Beschleunigungskräfte. Neben den, durch

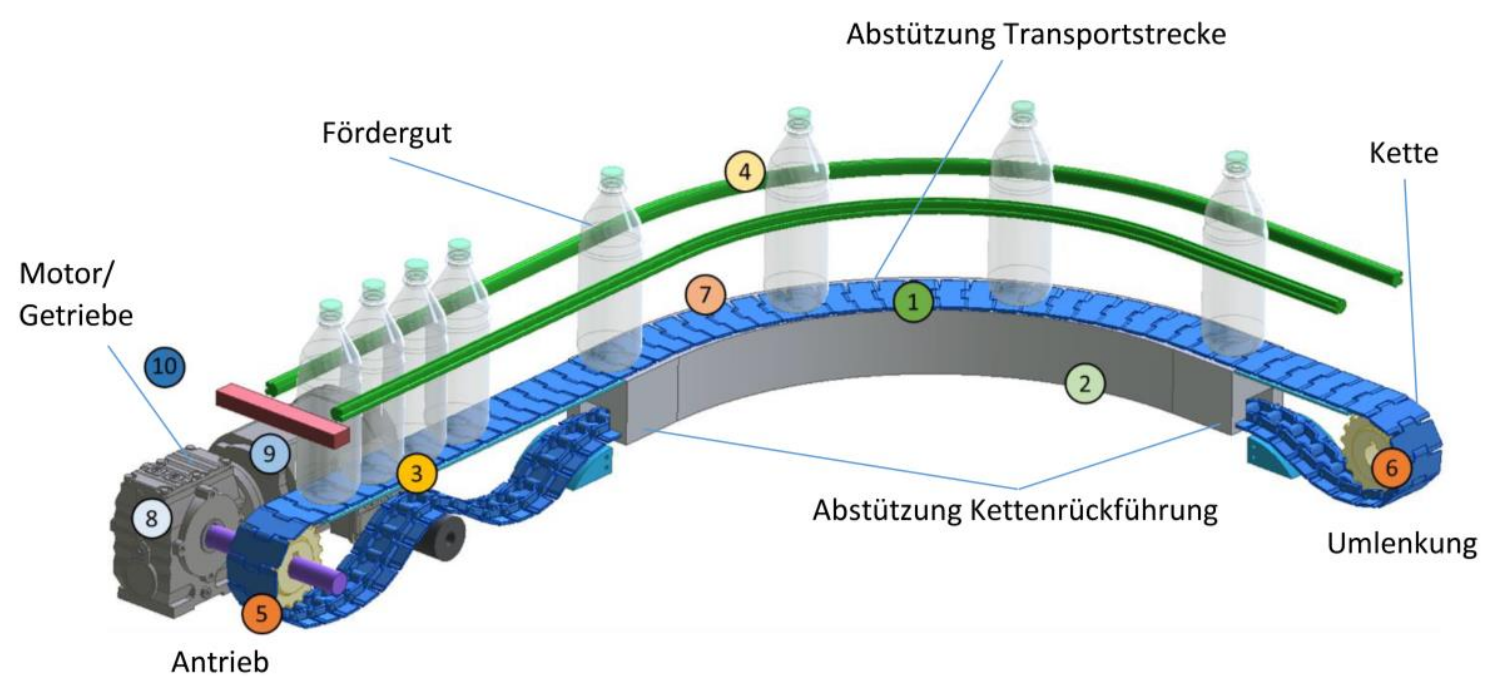

Abbildung 2: Verluste in einem Kettenförderer 
Tabelle 1: Verluste in einem Kettenförderer

\begin{tabular}{cl}
\hline Nr. & Bezeichnung \\
\hline 1 & Reibung Scharnierbandkette - Abstützung im Obertrum (Transportbereich) \\
2 & Reibung Scharnierbandkette - Abstützung im Untertrum (Kettenrückführung) \\
3 & Reibung Scharnierbandkette - Transportgut im Staubetrieb \\
4 & Reibung Transportgut - Seitenführung \\
$5 / 6$ & Reibung an Antrieb und Umlenkung (Lagerreibung, Schwenkbewegung \\
\hline 7 & der Kettengelenke, Reibung Kette - Kettenrad usw.) \\
\hline 8 & Wirkungsgradverlust Getriebe \\
9 & Wirkungsgradverlust Motor/Motorregelung \\
10 & Grundverbrauch der Peripherie (Netzteil, Lüfter, Steuerung usw.) \\
\hline
\end{tabular}

die Bewegung des Fördermittels entstehenden, Reibungsverlusten treten zudem auch Verluste auf, die auf das Antriebssystem zurückzuführen sind. Zur Verdeutlichung der Verluste eines Förderers sind in Abbildung 2 und Tabelle 1 alle Verlustanteile am Beispiel eines Scharnierbandkettenförderers in der Getränkeindustrie veranschaulicht.

\subsection{Gleitreibungsverluste}

Wie eingangs beschrieben, stellt das Reibungsverhalten aller tribologisch beanspruchten Komponenten den maßgeblichen Einflussfaktor auf die vom Motor aufzubringende Antriebsleistung dar. Die Reibungsverluste werden dabei insbesondere durch den Gleitkontakt der Kette zum Führungssystem beeinflusst. Bei der Berechnung der Kettenzugkraft eines ebenen Förderers werden grundsätzlich gerade Förderabschnitte und horizontale Gleitkurven unterschieden. In den geraden Förderabschnitten wirkt lediglich die Masse des Transportgutes im Kontakt zwischen Tragplatte und Gleitschiene, wohingegen in horizontalen Kurven zusätzlich eine Radialkraftkomponente zwischen Zugelement und Gleitschiene zu verzeichnen ist. In Gleitkurven ist daher, im Gegensatz zu geraden Abschnitten, ein exponentieller Anstieg der Kettenzugkraft zu verzeichnen [5]. Zusätzliche Verluste, die zu einer Erhöhung der Kettenumfangskraft führen, sind bspw. die Reibung zwischen Transportgut und Kette im Staubetrieb, die Seitenreibung zwischen Transportgut und Seitenführung aber auch Reibung zwischen den einzelnen Kettenelementen während eines Kurvenschwenks.

\subsection{Antriebskomponenten}

\section{Drehstrom-Asynchronmaschinen}

Asynchronmotoren sind in den industriellen Antrieben die am häufigsten verwendeten elektrischen Antriebe. Sie zeichnen sich durch einen einfachen und robusten Aufbau, große Betriebssicherheit, geringen wartungsaufwand und einen niedrigen Preis aus [6]. Drehstrom-Asynchronmotoren arbeiten im Teillastbereich nach Abbildung 3 mit wesentlich schlechteren Wirkungsgraden als bei Nennlast. Der sogenannte Teillastfaktor (TLF) ist dabei als Verhältnis der effektiv abgegebenen mechanischen Leistung und der Motor- 


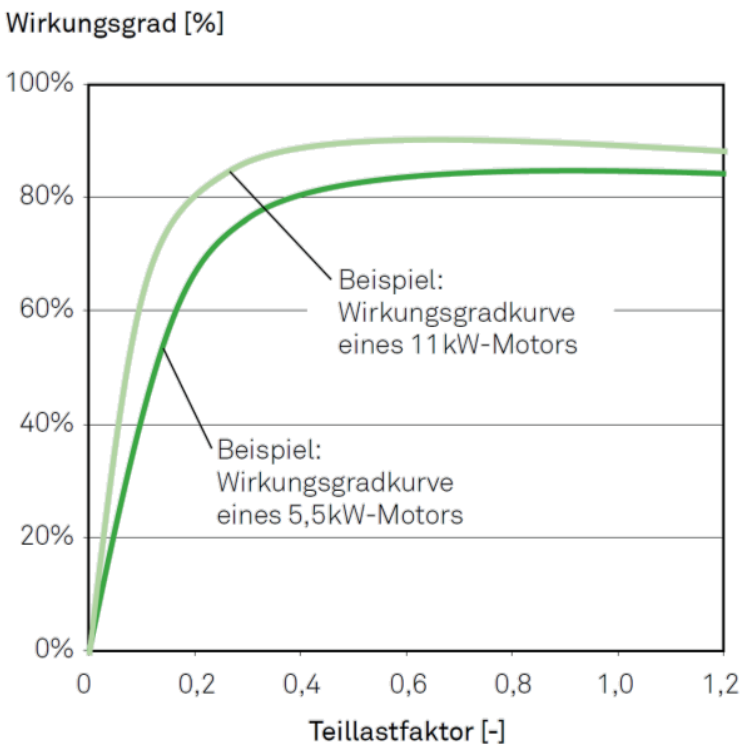

Abbildung 3: Wirkungsgrad verschiedener Motorgrößen in Abhängigkeit des Teillastfaktors [8]

nennleistung definiert. Daneben weisen Motoren mit kleineren Nennleistungen allgemein ein niedrigeres Wirkungsgradniveau auf [7]. Gerade in der Fördertechnik kommen kleinere Elektromotoren in großer Zahl zum Einsatz, die häufig im Teillastbereich betrieben werden. Die Reduzierung von Wirkungsgradverlusten birgt somit großes Energieeinsparungspotential.

\section{Frequenzumrichter}

Die Drehzahlen von Drehstrom-Asynchronmaschinen sind stark an die durch die Taktfrequenz und Polzahl festgelegte Synchrondrehzahl gebunden. Durch eine variable Speisefrequenz - generiert durch einen Frequenzumrichter (FU) - lässt sich dieser Nachteil jedoch ausgleichen. Frequenzumrichter erzeugen aus der Wechselspannung und der Frequenz $(50 \mathrm{~Hz})$ des Netzes eine Wechselspannung zur Speisung von elektrischen Maschinen, welche in Frequenz und Amplitude veränderbar ist [9]. Moderne Frequenzumrichter bestehen aus drei Komponenten einem Gleichrichter, einem Gleichspannungszwischenkreis sowie einem Wechselrichter. Der netzseitig steuerbaren Gleichrichter wandelt dabei die dreiphasige Netzspannung in eine Gleichspannung um. Durch den Wechselrichter wird aus der Gleichspannung im Anschluss wieder eine dreiphasige Wechselspannung mit steuerbarer Frequenz generiert [10]. Genau wie bei DrehstromAsynchronmotoren sinken auch bei Frequenzumrichtern die Wirkungsgrade im Teillastbereich zum Teil erheblich [9].

\section{Getriebe}

In der Stückgutfördertechnik kommen vorwiegend Motor-Getriebe-Kombinationen mit Untersetzungsgetrieben zum Einsatz [6]. Eine häufig eingesetzte Variante ist das Schneckengetriebe, welches es erlaubt große Übersetzungsverhältnisse in nur einer Getriebestufe $\mathrm{zu}$ realisieren. Als Orientierungswerte werden für die Wirkungsgrade von Schneckengetriebe 0,2...0,97 angeben. Grundlegend gilt je höher die Übersetzung desto niedriger der erreichbare Wirkungsgrad [11]. Daneben kann auch bei Schneckengetrieben 
eine ausgeprägte Abhängigkeit der Effizienz zum Lastgrad und zur Eingangsdrehzahl festgestellt werden. Auch hier nimmt der Wirkungsgrad bei Teillast und bei Drehzahlen unterhalb der Nenndrehzahlen ab [12].

\section{Experimentelle Bestimmung des Antriebswirkungsgrades}

\subsection{Versuchsdurchführung}

Die Bestimmung der Wirkungsgrade einer gewählten Antriebskombination erfolgte an einem Motorenversuchsstand nach Abbildung 4. Dabei überträgt die Antriebseinheit das Drehmoment $M$ über eine Drehmomentmesswelle auf eine Magnetpulverbremse. Diese stellt ein geregeltes Bremsmoment zur Verfügung, wodurch der Motor in verschiedenen Lastbereichen geprüft werden kann. Durch zusätzliche Messung der Wellendrehzahl $n$ kann die abgegebene mechanische Leistung der Antriebseinheit mit

$$
P_{\text {mech }}=M \cdot \omega=M \cdot 2 \pi \cdot n
$$

berechnet werden.

Zur Ermittlung der Effizienz der Antriebseinheit wurden jeweils mehrere Versuchsreihen mit unterschiedlichen Taktfrequenzen des Frequenzumrichters durchgeführt. Der Motor wurde bei jeder Geschwindigkeit mit schrittweise steigenden Bremsmomenten belastet und die dabei aufgenommene elektrische Wirkleistung direkt mit einem digitalen Leistungsmessgerät vor dem Frequenzumrichter gemessen. Der Wirkungsgrad im jeweiligen Lastbereich kann so nach Gleichung (1) als Quotient aus der abgegebenen mechanischen Leistung und der aufgenommenen elektrischen Wirkleistung berechnet werden. Der dargestellte Versuchsaufbau macht deutlich, dass es sich bei der gemessenen Effizienz um einen Gesamtwirkungsgrad aus Asynchronmaschine, Schneckengetriebe sowie Frequenzumrichter handelt.

Die Untersuchungen wurden an einer repräsentativen Motor-Getriebe-Kombination mit integriertem Frequenzumrichter mit den in Tabelle 2 dargestellten Leistungsdaten durchgeführt. Ebenfalls in Tabelle 2 sind weitere Versuchsparameter aufgezeigt.

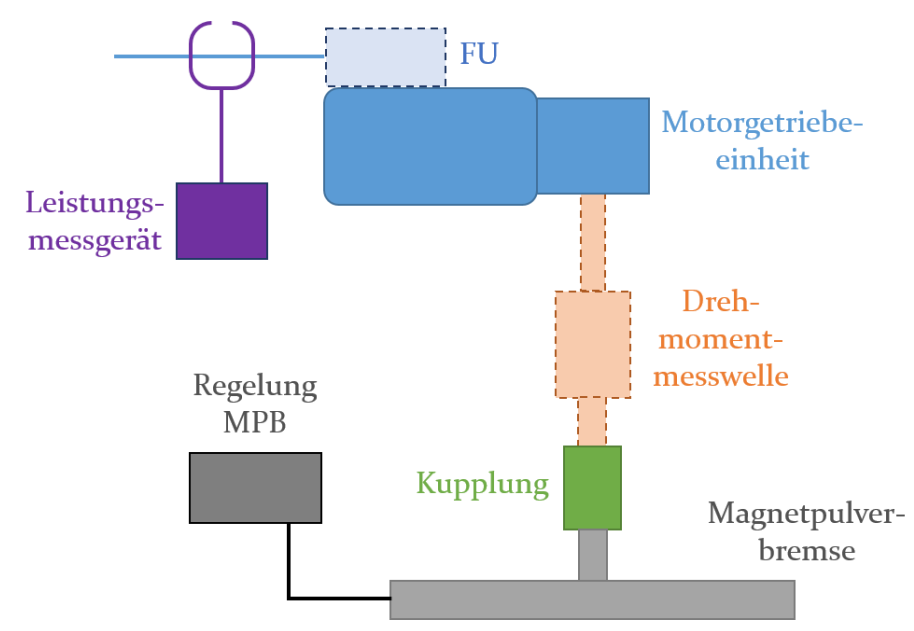

Abbildung 4: Schematischer Aufbau des Motorenversuchsstandes 
Tabelle 2: Kenndaten des gewählten Antriebssystems und Versuchsparameter

\begin{tabular}{|c|c|}
\hline $\begin{array}{cl}\text { Asynchrongetriebemotor mit F } \\
\text { - } & \text { Hersteller } \\
\text { - } & \text { Bezeichnung } \\
\end{array}$ & $\begin{array}{l}\text { SEW } \\
\text { SH57/T DRE90L4/MM22/LN }\end{array}$ \\
\hline $\begin{array}{l}\text { Leistungsdaten bei } 100 \mathrm{~Hz}[13] \\
\text { - Nennleistung: } \\
\text { - Nenndrehmoment: } \\
\text { - Nenndrehzahl: } \\
\text { - } \quad \text { Getriebeübersetzung: }\end{array}$ & $\begin{array}{l}P_{\text {Nenn }}=2,2 \mathrm{~kW} \\
M_{\text {Nenn }}=153 \mathrm{Nm} \\
n_{\text {Nenn }}=2.900 / 1171 / \mathrm{min} \\
i=24,77\end{array}$ \\
\hline Frequenzstufen am Umrichter & 12 bis $100 \mathrm{~Hz}$ in 6 Lastststufen \\
\hline Bremsmoment & 0 bis $200 \mathrm{Nm}$ in 6 Lastststufen \\
\hline
\end{tabular}

\subsection{Auswertung}

Bei den Messungen wurde der erwartete lineare Zusammenhang zwischen der eingestellten Umrichterfrequenz und der Drehzahl am Getriebeausgang festgestellt. Zudem ergeben sich die in Abbildung 5 abgebildeten charakteristischen Wirkungsgradkennlinien.

Die besten Wirkungsgrade werden im 100 Hz-Betrieb (blaue Kurve) erreicht, der Maximalwert wurde dabei mit $\eta_{\max }=0,71 \mathrm{im}$ Bereich der theoretischen Motornennlast von 179,6 Nm und 117 1/min (ohne Getriebeverluste) am Getriebeausgang gemessen. Aus Abbildung 5 geht weiter hervor, dass der Wirkungsgrad nicht allein von der Lastabgabe des Motors abhängt, sondern - in Kombination mit einem Frequenzumrichter - außerdem stark von der Taktfrequenz bzw. der Motordrehzahl beeinflusst wird. So ist der Gesamtwirkungsgrad der Antriebskomponenten bei geringen Drehzahlen deutlich schlechter als bei hohen Drehgeschwindigkeiten.

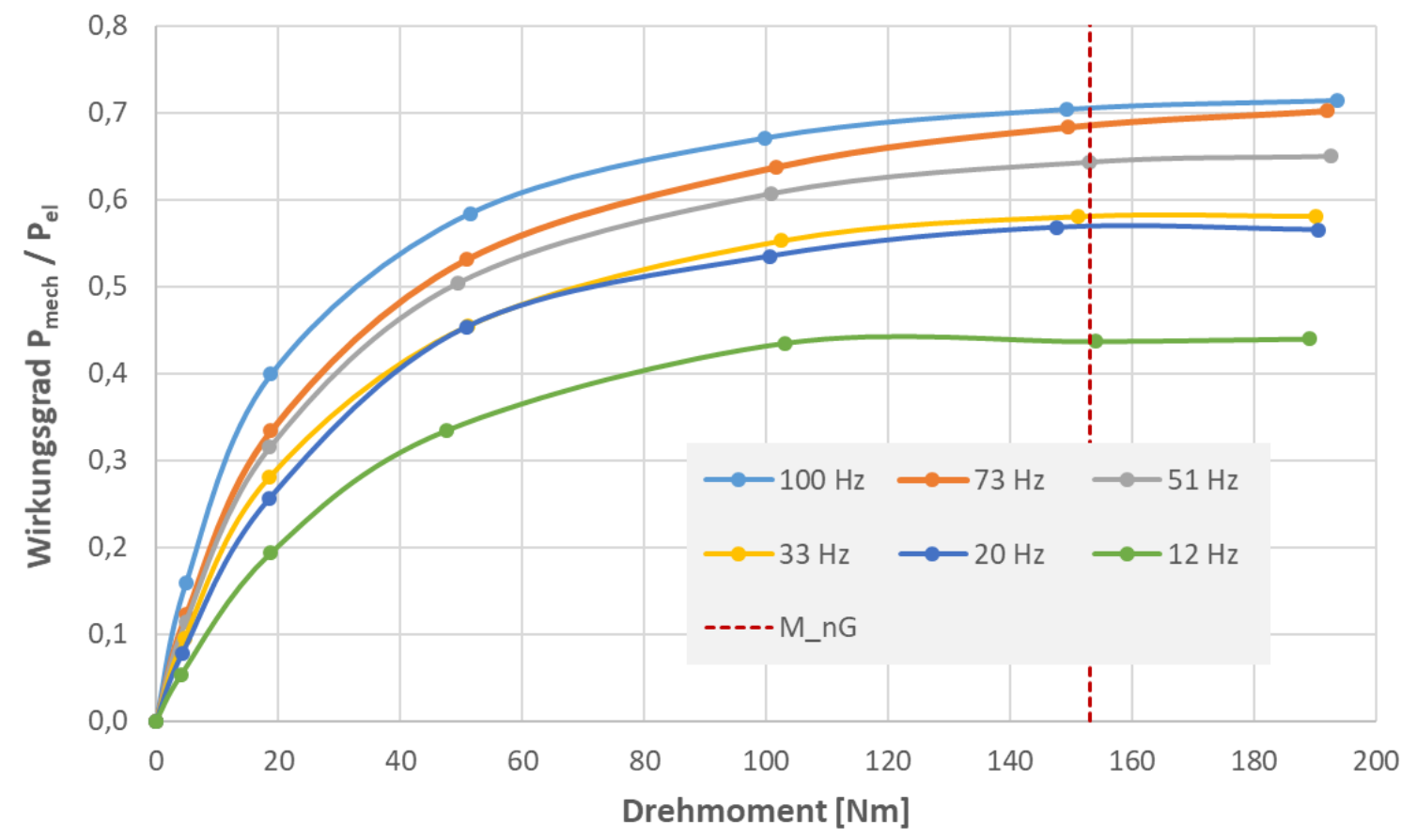

Abbildung 5: Wirkungsgrade der untersuchten Antriebseinheit in Abhängigkeit vom Lastmoment und der Taktfrequenz des Umrichters sowie Nennmoment $M_{n G}$ am Getriebeausgang bei $T L F=1 \mathrm{und} 100 \mathrm{~Hz}$ 


\section{Analytische Betrachtungen zur Effizienz des Antriebs}

\subsection{Beispielfördersystem}

Die experimentell gewonnenen Ergebnisse aus den Motorversuchen sollen im Folgenden auf einen bespielhaft gewählten Gleitkettenförderer mit Scharnierbandketten bezogen werden. Die einzelnen Leistungsanteile werden zu diesem Zweck analytisch berechnet. Ziel ist es, die Verluste des Antriebssystems im Kontext der Gesamtverluste eines Stetigförderers exemplarisch abzubilden, gegenüberzustellen und Handlungsempfehlungen zur Verbesserung der Effizienz abzuleiten.

Bei dem gewählten Beispiel handelt es sich um einen Scharnierbandkettenförderer mit typischen Streckenelementen. Das Layout nach Abbildung 6 beinhaltet zwei Geraden sowie eine $90^{\circ}$-Horizontalkurve. Die für die folgenden Analysen benötigten Daten basieren auf den in Tabelle 3 gezeigten Ausgangsgrößen.

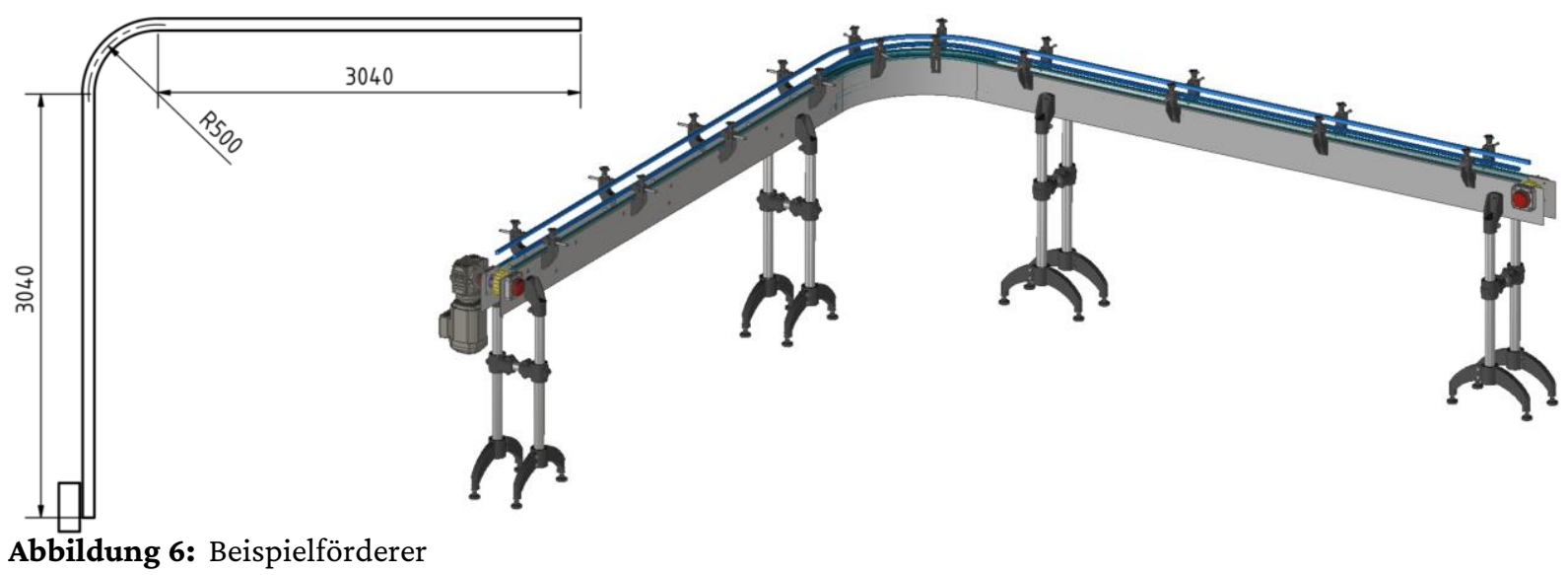

\begin{tabular}{ll} 
Tabelle 3: Kenndaten des Beispielförderers; Motordaten vgl. Abschnitt 4.1 \\
\hline Streckenlayout & \\
• $\quad$ Länge der Geradabschnitte: & $L=3,04 \mathrm{~m}$ \\
- $\quad$ Radius der Gleitkurve: & $R=500 \mathrm{~mm}$ \\
\hline Asynchrongetriebemotor & $P_{N G}=2,2 \mathrm{~kW}$ \\
- Nennleistung am Getriebeausgang: & $M_{N G}=153 \mathrm{Nm}$ \\
- Nenndrehmoment am Getriebeausgang: & $n_{N G}=1171 / \mathrm{min}$ \\
• Nenndrehzahl am Getriebeausgang: & \\
\hline Förderkette (Scharnierbandkette, Stahl) & $q_{Z}=3 \mathrm{~kg} / \mathrm{m}$ \\
- $\quad$ spezifische Kettenmasse: & $\mu_{Z S}=0,3$ \\
• $\quad$ Reibwert Kette vs. Führungsschiene: &
\end{tabular}

\subsection{Berechnung der benötigten Antriebsleistung}

Um die mechanische Antriebsleistung $P_{\text {mech }}$ des Scharnierbandkettenförderers analytisch berechnen $\mathrm{zu}$ können, muss zunächst die Umfangskraft $F_{U}$ ermittelt werden, die bei ungespannten Kettenförderern der maximalen Kettenzugkraft entspricht. Hierzu erfolgt eine Segmentierung des Fördersystems in spezifische Abschnitte wie Geraden, Kurven, 
Staustrecken usw. Die maximale Kettenkraft ergibt sich dabei durch Aufsummierung der Kräfte $F_{A b}$ aller Einzelsegmente in Bewegungsrichtung der Kette. Die Berechnung wird im Auslauf der Kette aus dem Kettenrad begonnen. In diesem als Kettensack bezeichneten Bereich ist die Kettenzugkraft annähernd $0 \mathrm{~N}$. Die ermittelte Kettenkraft des ersten Abschnittes geht dann als Startwert in die Berechnung des nachfolgenden Abschnittes ein [5]:

$$
F_{n}=F_{n-1}+F_{A b}
$$

Somit ergibt sich die Umfangskraft $F_{U}$ als maximale Kettenzugkraft beim Einlauf der Kette in die Antriebseinheit. Ein typischer Zugkraftverlauf des Beispielförderers ist in Abbildung 7 dargestellt. Es wird deutlich, dass die Kette im Untertrum (Kettenrückführung) vergleichs weise gering beansprucht wird und die Zugkraft erst im Bereich der Transportstrecke in Abhängigkeit von der Höhe der Beladung und den Reibwerten deutlich ansteigt. Ebenso ist der bereits oben beschriebene, gegenüber den Geradabschnitten deutlich stärkere Zugkraftanstiegt im Gleitbogen erkennbar, der durch die zusätzliche radiale Abstützung verursacht wird.

Durch Multiplikation der Umfangskraft $F_{U}$ mit der Kettengeschwindigkeit $v_{K}$ ergibt sich die erforderliche mechanische Antriebsleistung zum Betreiben des Förderers mit

$$
P_{\text {mech }}=F_{U} \cdot v_{K}
$$

sowie mit einer bekannten (gemessenen) elektrischen Wirkleistung $P_{e l}$ der Wirkungsgrad

$$
\eta_{\text {Ges }}=\frac{P_{\text {mech }}}{P_{e l}}
$$

des Antriebssystems. Wie bereits erwähnt ist zu beachten, dass es sich dabei um den Gesamtwirkungsgrad des Förderers handelt, der sich im betrachteten Beispiel aus den Verlustanteilen von Motor, Schneckengetriebe und Frequenzumrichter zusammensetzt.

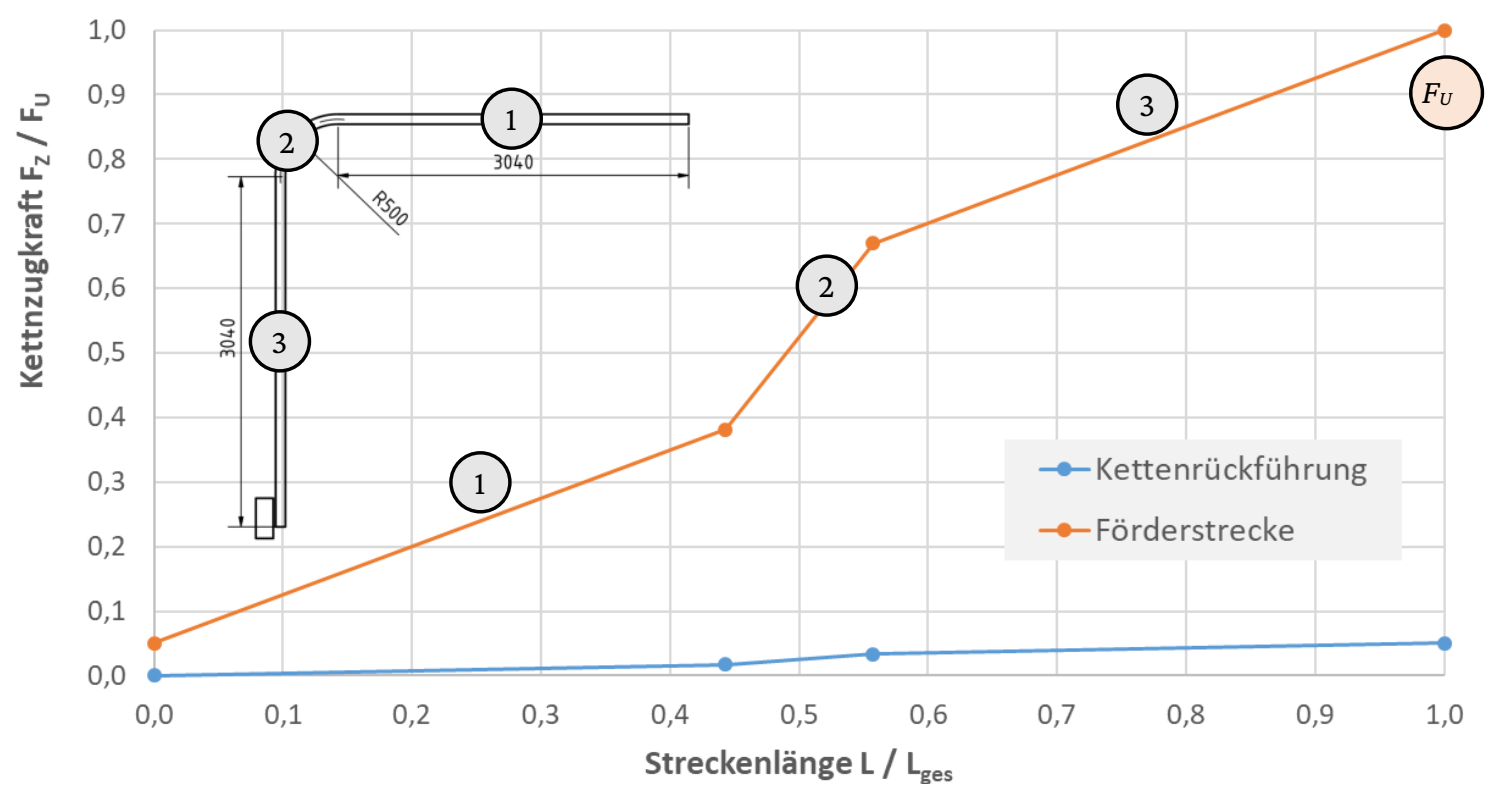

Abbildung 7: Exemplarischer Kettenzugkraftverlauf des Beispielförderers mit Umfangskraft $F_{U}$ : erster Geradabschnitt (1), Gleitbogen (2) sowie zweiter Geradabschnitt incl. Antrieb (3) der Förderstrecke 


\subsection{Analyse der Einflussparameter}

\section{Einfluss der Umrichterfrequenz und der spezifischen Gutmasse}

Anhand der verfügbaren Berechnungsgrundlagen sowie der experimentell ermittelten Leistungsdaten des Getriebemotors werden im Folgenden die Wirkungen einiger Einflussparameter diskutiert.

Drehstromasynchronmaschinen arbeiten nur in ihrem Nennpunkt stabil und mit akzeptablen Wirkungsgraden. Aus diesem Grund soll sich in einer ersten Betrachtung an der Nennleistung des Antriebsmotors orientiert werden. Bei dem hier gewählten Beispielförderer wird der Motornennpunkt nur bei einer sehr hohen Beladung von rund $90 \mathrm{~kg} / \mathrm{m}$ und einer Kettengeschwindigkeit von $46,5 \mathrm{~m} / \mathrm{min}$ (100 Hz) erreicht. Nach der experimentell ermittelten Effizienz aus 4.2 ergibt sich so ein Gesamtwirkungsgrad von 0,71 bei Nennlast (Abbildung 8, roter Datenpunkt).

Wird die spezifische Gutmasse gesenkt, führt dies erwartungsgemäß zu einer Verschlechterung des Wirkungsgrades. Während dieser bei $51 \mathrm{~Hz}$ noch $64,5 \%$ beträgt, werden bei $12 \mathrm{~Hz}$ nur noch $44 \%$ erreicht. Neben den Einfluss der Beladung wird aus Abbildung 8 ebenso die starke Abhängigkeit zur Umrichterfrequenz deutlich. Eine Reduzierung der Taktfrequenz, ergo der Kettengeschwindigkeit, vermindert den Wirkungsgrad des Förderers ebenfalls erheblich.

In Abbildung 9, links sind ausgehend von der Nennleistung noch einmal die berechneten Leistungsanteile bei konstanter spezifischer Gutmasse von $90 \mathrm{~kg} / \mathrm{m}$ gezeigt. Erwartungsgemäß sind die Reibverluste im Obertrum dabei am größten, die Verluste des Antriebssystems betragen entsprechend der gemessenen Effizienz ca. $29 \%$ der berechneten elektrischen Wirkleistung.

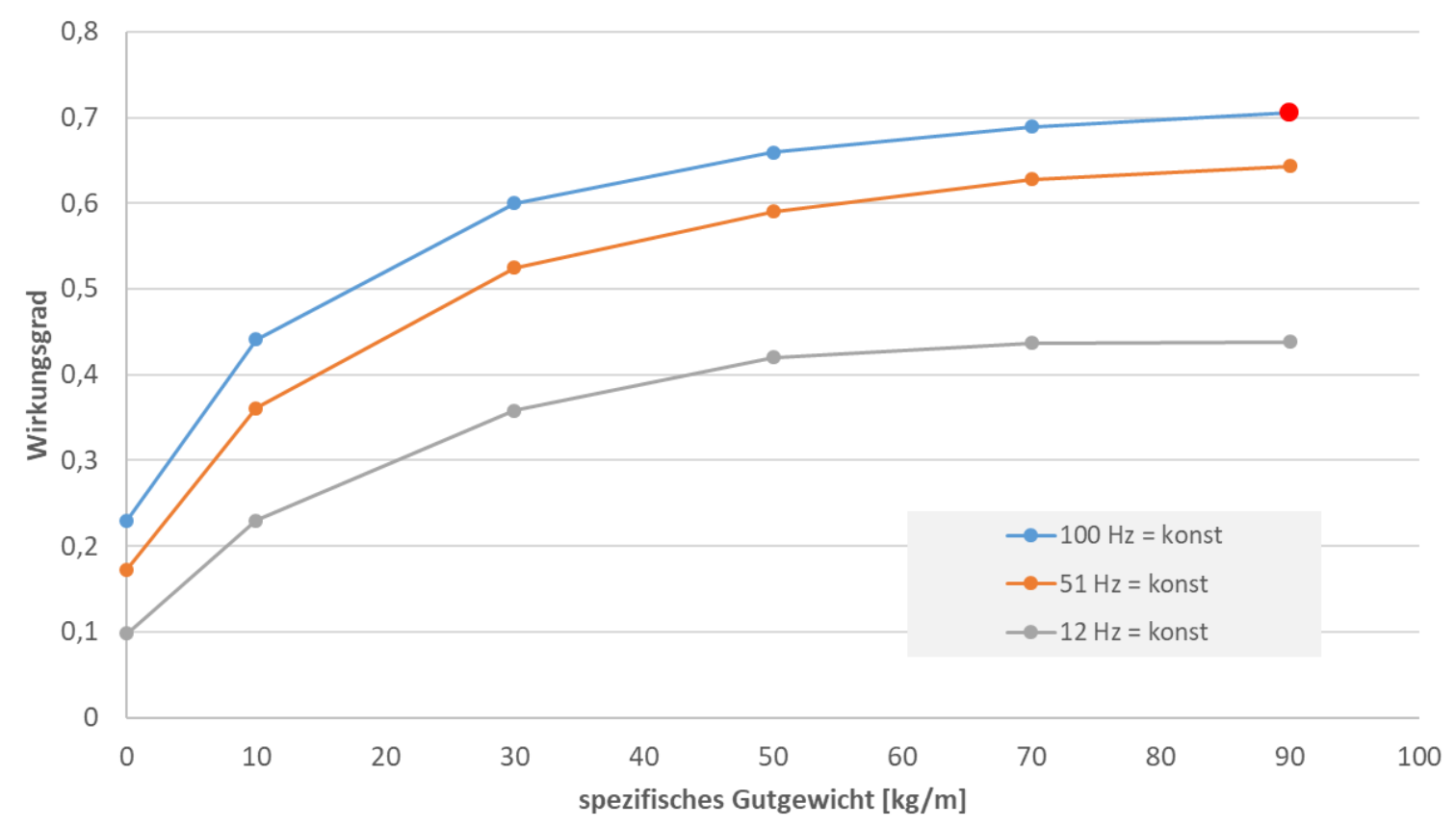

Abbildung 8: Wirkungsgrad des Beispielförderers in Abhängigkeit der Beladung und der Taktfrequenz; roter Datenpunkt entspricht Nennbetrieb des Antriebsmotors 


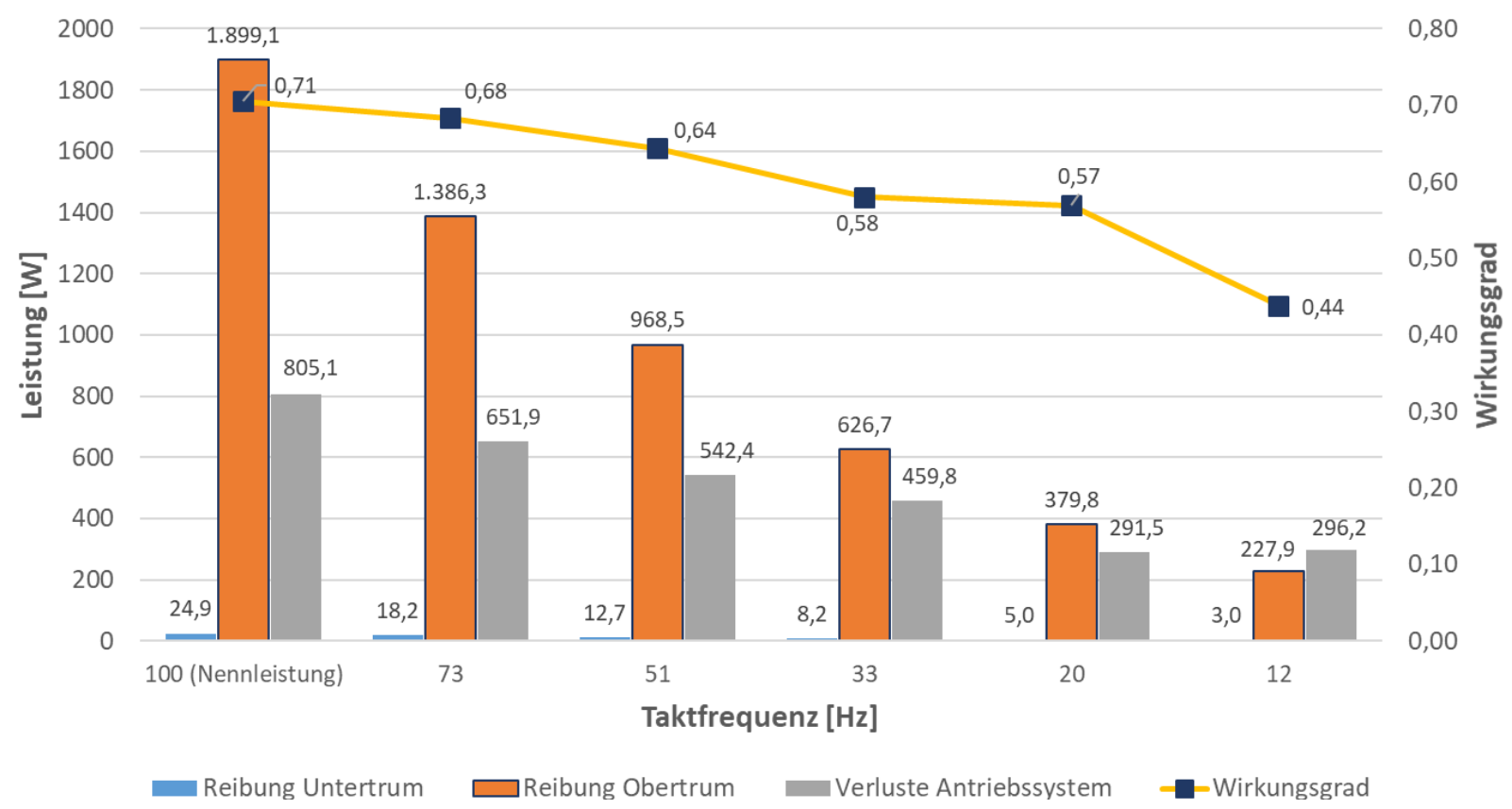

Abbildung 9: Leistungsaufnahme des Beispielförderers bei verschiedenen Taktfrequenzen

Wird die Umrichterfrequenz reduziert, vermindern sich folglich auch die einzelnen Leistungsanteile. Aufgrund des linearen Zusammenhangs zwischen Umfangskraft und Kettengeschwindigkeit nach Gleichung (4) nehmen auch die Anteile der mechanischen Leistung durch eine Verringerung der Taktfrequenz gleichmäßig ab. Dementgegen nehmen die Verluste des Antriebssystems zu. Bei einer Speisefrequenz von $12 \mathrm{~Hz}$ übersteigen diese sogar die mechanischen Verluste

\section{Einfluss des Reibwertes}

Viele Bestrebungen von Fördersystembetreibern zielen auf eine Verbesserung der Reibverhältnisse zwischen Gleitkette und Gleitelementen ab. Die Substitution von gebrauchten Gleitelemente und -ketten zu bspw. additivierten Kunststoffen verbessern den Reibwert deutlich. Um das gesamte Energieeinsparungspotential einer solchen Maßnahme im Kontext des Gesamtsystems Gleitkettenförderer zu nutzen, macht dies allerdings nur Sinn, wenn gleichzeitig auch eine Optimierung des Antriebsystems vorgenommen wird. Abbildung 10 verdeutlicht diesen Sachverhalt und stellt die Leistungsanteile für verschiedene Reibwerte bei einer spezifischen Gutmasse von 21,5 kg Gutmasse pro Meter und einer Taktfrequenz von $100 \mathrm{~Hz}$ gegenüber. Dabei wird deutlich, dass die aufgenommene Wirkleistung des Beispielförderers mit 2,2 kW-Motor durch eine Reduktion des Reibwertes von $978 \mathrm{~W}$ bei 0,3 auf $494 \mathrm{~W}$ bei 0,1 (Abbildung 10) zwar sinkt. Gleichzeitig verringert sich jedoch auch der Wirkungsgrad von 0,55 auf 0,32.

Im Gegenzug verbessert sich die Effizienz des Systems bei einem Reibwert von 0,5 auf einen Wert von $61 \%$. Während sich durch höhere Reibwerte die Reibleistungen im Ober - und Untertrum erwartungsgemäß exponentiell erhöhen sinkt folglich der Anteil an Verlusten im Antriebssystem. 


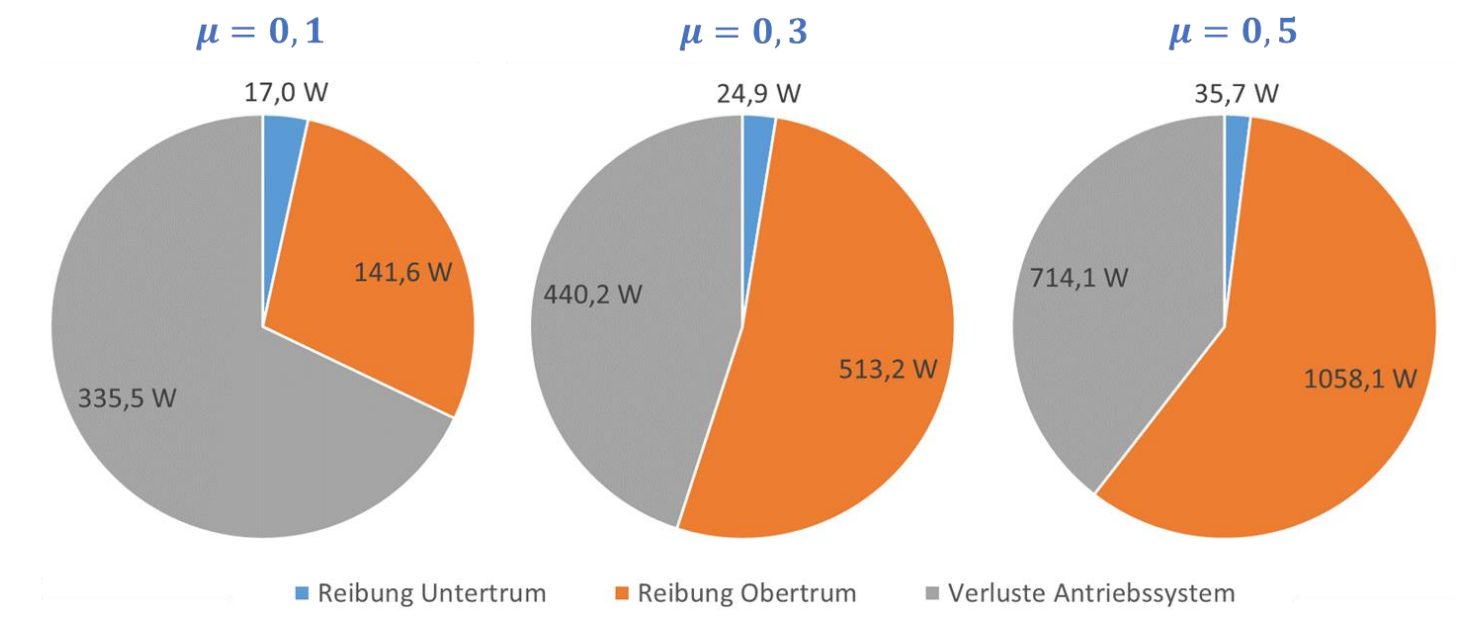

Abbildung 10: Einfluss der Reibwertes auf die Leistungsanteile (Reibwert: links 0,1, Mitte 0,3, rechts 0,5)

\section{Einfluss des Fördermassenstroms}

Wie in den vorigen Abschnitt beschrieben, führen Senkungen der spezifischen Gutmasse oder des Reibwertes bei konstanten Motordrehzahlen zu einem geringeren, vom Antriebsmotor aufzubringenden Drehmoment, was wiederum einen schlechteren Wirkungsgrad entlang der jeweiligen Taktfrequenzkurve (Abbildung 5) zur Folge hat. Verringerungen der Drehzahl unterhalb der Nenndrehzahl führen dazu, dass der Motor in einem anderen Wirkungsgradniveau arbeitet. Eine Effizienzverschlechterung ist dabei ebenfalls $\mathrm{zu}$ verzeichnen.

In der Praxis kann die Verringerung der spezifischen Gutmasse jedoch nicht ohne eine Anpassung der Fördergeschwindigkeit einhergehen. Ziel des Fördertechnikanwenders ist die Bereitstellung eines konstanten Fördermassenstroms $\dot{m}$ für nachfolgende Maschinen und Anlagen. Der Fördermassenstrom ist definiert nachfolgender Gleichung, wobei $q_{G}$ der Gutmasse pro Meter Förderstrecke entspricht.

$$
\dot{m}=q_{G} \cdot v_{K}
$$

Demzufolge muss bei einer Verringerung der spezifischen Gutmasse die Kettengeschwindigkeit erhöht werden um den Fördermassenstrom konstant zu halten. Die nachfolgende Abbildung zeigt die Effizienz des Förderers in Abhängigkeit der Taktfrequenz bei Massenströmen von $0 \mathrm{~kg} / \mathrm{min}$ (Leerlauf) bis $2000 \mathrm{~kg} / \mathrm{min}$.

Auffällig ist der relativ konstante Verlauf der Wirkungsgrade bei jedem Massenstrom über der Speisefrequenz. Die einzelnen Kurven führen bei kleinen Drehzahlen zu keinem nennenswerten Abfall der Effizienz. Erklären lässt sich dieses Verhalten durch die gleichzeitige Erhöhung der Gutmasse, welche zu einem größeren Drehmoment und somit zu einer höheren Effizienz führt. Bei konstanten Fördermassenströmen hat die Taktfrequenz im berechneten Beispiel mit 2,2 kW-Motor demzufolge nur wenig Einfluss. 


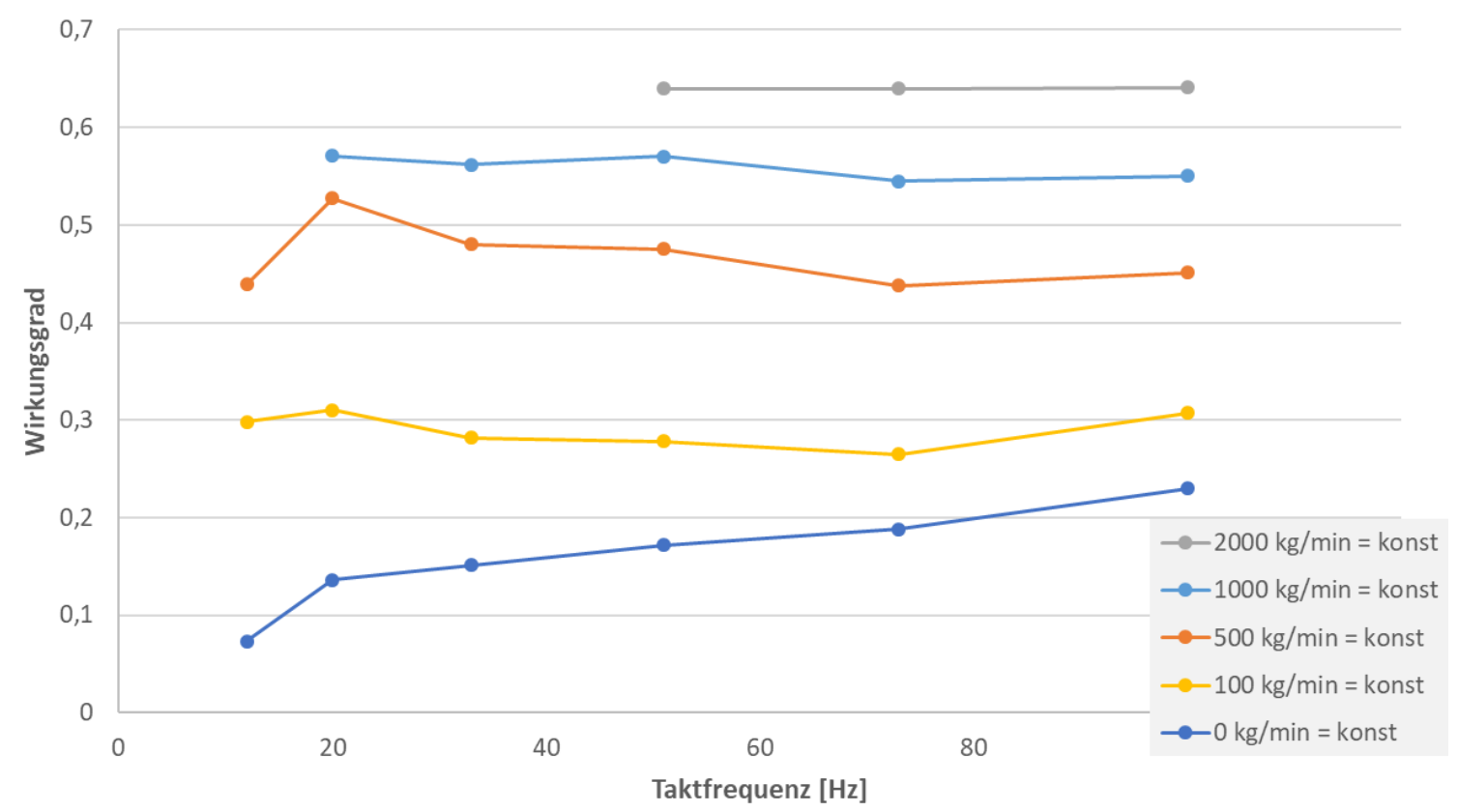

Abbildung 11: Wirkungsgrade des Beispielförderers bei verschiedenen konstanten Fördermasseströmen in Abhängigkeit der Taktfrequenz

\section{Zusammenfassung und Ausblick}

Der vorliegende Beitrag befasst sich mit Verlusten in Kettenförderern, welche den Gesamtwirkungsgrad des Systems maßgeblich beeinflussen. Vor allem das Antriebssystem lag im Fokus dieser Untersuchungen, da es - je nach Auslegung - zu einem nicht unerheblichen Teil zu Leistungsverlusten beitragen kann. Experimentelle Untersuchungen eines Asynchron-Getriebe-Motors mit Frequenzumrichter haben dabei einen starken Einfluss sowohl der aufgebrachten Last, als auch der Umrichterfrequenz auf die Effizienz der Antriebskombination gezeigt. Die experimentell gewonnenen Daten wurden dann auf einen beispielhaft gewählten Scharnierbandkettenförderer bezogen, verschiedene Einflüsse auf die Gesamteffizienz diskutiert und Abhängigkeiten auf die elektrische Wirkleistungsaufnahme dargestellt. Um die Verluste von Asynchron-Getriebe-Motoren möglichst gering zu halten müssen diese speziell auf die Förderaufgabe und das Fördersystem abgestimmt sein. Die gängige Praxis einer Überdimensionierung sollte vor allem im Hinblick auf die Reduktion von Umweltschädigungen durch Klimagasemissionen überdacht werden. Einzelne Optimierungsmaßnahmen, beispielsweise die Verbesserung des Reibwertes durch Substitution von Gleitelementen sind sinnvoll, müssen jedoch mit einer Optimierung des Antriebes einhergehen um das gesamte Energieeinsparungspotential voll auszuschöpfen.

In den nächsten Schritten sollen die Antriebskomponenten, welche in diesem Beitrag als System betrachtet wurden, einzeln analysiert werden. Ziel ist es den Asynchronmotor, das Getriebe und den Frequenzumrichter in Bezug auf ihre Verluste durch experimentelle Untersuchungen individuell zu bewerten. 


\section{Danksagung}

Dieses Projekt wird gefördert durch die Röchling Stiftung.

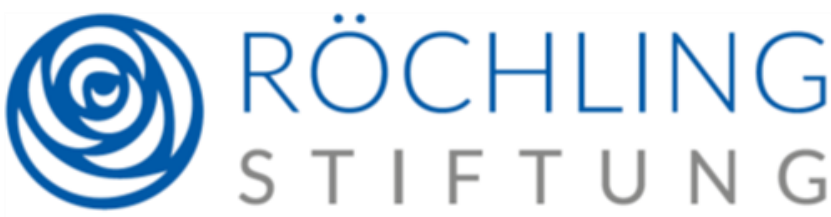

\section{Quellen}

[1] N.N.: Internetpräsentation, Bosch Rexroth AG, https://www.boschrexroth.com/de/de/produkte/ produktgruppen/montagetechnik/index, Zugriff 14.10.2020

[2] N.N.: SynCo. Krones AG, https://www.krones.com/media/downloads/synco_de.pdf, Zugriff 14.10.2020

[3] Schumann, A., Weise, S., Sumpf, J.: Oberflächenstrukturen zur Reibungs - und Verschleißreduzierung von Kunststoff-Reibpaarungen in Förderanlagen. Tribologie + Schmierungstechnik, 59. Jahrgang, Seite 19-23 $(01 / 2012)$

[4] Weise, S., Schumann, A., Sumpf, J.: Charakterisierung schmierungsfreier Kunststoff-KunststoffReibpaarungen. Tagungsband TECHNOMER 2011, Chemnitz, Eigenverlag, 2011, S.84, ISBN: 978-3-939382-10-2

[5] Auerbach, P.: Zur Beanspruchung und Lebensdauer raumgängiger Gleitketten aus Kunststoff. Dissertation, Technische Universität Chemnitz, 2006. https://nbn-resolving.org/urn:nbn:de:swb:ch1-200600396

[6] VDI 4421:2000: Antriebstechniken in der Stückgutfördertechnik - Übersicht und Auswahl. Berlin: BeuthVerlag 2000.

[7] Rudolf M., Wagner U.: Energieanwendungstechnik: Wege und Techniken zur effizienten Energienutzung. Berlin: Springer, 2008.

[8] N.N.: Merkblatt 9: Lastfaktor. www.topmotors.ch, Zürich, 10.2014, https:/www.topmotors.ch/sites/default/files/2018-08/D_MB_09_Teillastfaktor.pdf, Zugriff 14.10.2020

[9] Brunner C., Tieben R., Omlin A.: Merkblatt 25: Frequenzumrichter. www.topmotors.ch, Zürich, 10.2014, https://www.topmotors.ch/sites/default/files/2018-08/D_MB_25_Frequenzumrichter.pdf, Zugriff 14.10.2020

[10] Beckert U.: Asynchronmotor: Stationäres Betriebsverhalten. Skriptum, TU Bergakademie Freiberg, Institut für Elektrotechnik, https://tu-freiberg.de/sites/default/files/media/institut-fuer-elektrotechnik12774/UBeckert_Scr/asm_stat_verhalten.pdf, Zugriff 14.10.2020

[11] Steinhilper W., Sauer B.: Konstruktionselemente des Maschinenbaus 2: Grundlagen von Maschinenelementen für Antriebsaufgaben. 7. Aufl. Berlin: Springer, 2012

[12] Radosavljević S., Stojanović B., Skulić D.: Determination of power losses in worm gear reducer. IOP Conf. Series: Materials Science and Engineering 393, 2018.

[13] SEW-Eurodrive GmbH \& Co. KG: Technische Daten und Maßblätter MOVIMOT ${ }^{\circledR}$ - Lieferbare MOVIMOT ${ }^{\circledR}$ Motorkombinationen, www.sew-eurodrive.com, Abruf 13.10.2020 